\title{
Hemin/G-quadruplex Complex as a Signal Generator for Electrochemical Assays of Bioanalytes Hisakage FUNABASHI*
}

\author{
Institute for Sustainable Sciences and Development, Hiroshima University, \\ 1-3-2 Kagamiyama, Higashihiroshima, Hiroshima 739-8511, Japan
}

* Corresponding author: hisafuna@hiroshima-u.ac.jp

\begin{abstract}
Single-stranded deoxyribonucleic acid (ssDNA) with guanine-rich sequences can form four-stranded structures so called G-quadruplex $(\mathrm{Gq})$, which binds hemin, an iron-containing porphyrin. The hemin/Gq is an electroactive complex and exhibits peroxidase activity, and thus the hemin/Gq complexes have attracted great attention as a signal generator. This short review surveys the use of the hemin/Gq complex as an electrochemical signal generator. Electrochemical methods for detecting the hemin/Gq complex, approaches to signal amplification, and targeting strategies are herein discussed. Compared with protein-based signal generators, hemin/Gq complexes have some advantages: a wide variety of ssDNA sequences with various chemical modifications are easy to obtain, they can be immobilized on an electrode easily, and they can be designed as desired to connect functional domains. Also there are many molecular-biological tools to handle them. Hemin/Gq complexes have been successfully used to detect bioanalytes ranging from low molecular weight compound to macromolecules such as proteins, specific nucleic acids, or living cancer cells. Such bioanalytes are critical to the investigation of cellular function. Thus, DNAbased probes that contain hemin/Gq as a signal generator are a promising tool for the electrochemical analysis of cellular function, offering a competent alternative to a conventional protein-based signal generator.
\end{abstract}

(C) The Electrochemical Society of Japan, All rights reserved.

Keywords : DNA-based Biosensor, DNazyme, G-quadruplex, Hemin

\section{Introduction}

A single-stranded deoxyribonucleic acid (ssDNA) with a guanine-rich sequence can form four-stranded structures so called G-quadruplex $(\mathrm{Gq})$ structure. ${ }^{1}$ The guanines forming a square planar in the Gq structure bind to hemin (Fig. 1), an electroactive ironcontaining porphyrin. The hemin/Gq complex enhances the heminbased peroxidation reaction, becoming a DNazyme that exhibits peroxidase activity. ${ }^{2,3}$ The development of analytical and handling techniques for DNA as a material have generated interest in hemin/ Gq complexes as signal generators for the detection of bioanalytes such as nucleic acids, proteins, and metabolites with colorimetric, fluorescent, and luminescent signals., ${ }^{2,4}$ A protein exhibiting peroxidase activity such as horse radish peroxidase (HRP) has been widely used for electrochemical detection as a signal generator.
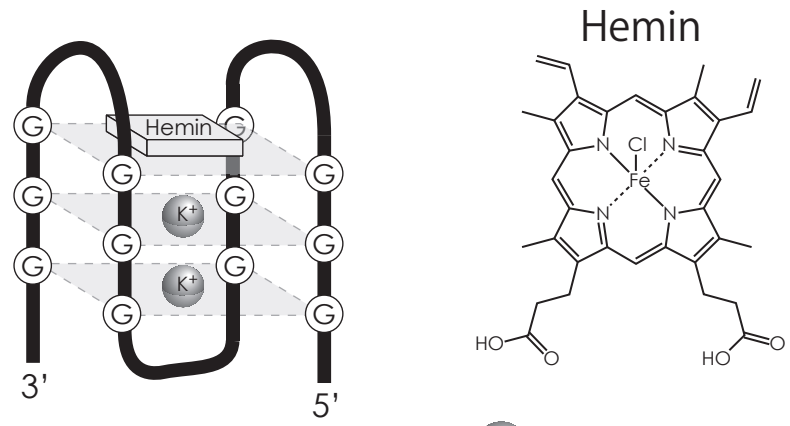

\section{Potassium ion}

Figure 1. An antiparallel-type complex is shown on the left side of the figure as an example of the hemin/Gq structure. Parallel- and hybrid-type complexes are also possible. The chemical structure of hemin is shown on the right side of the figure.
Therefore, the peroxidase activity of hemin/Gq complexes makes them a natural choice for signal generation in electrochemical analyses. Although it has been reported that the unit activity of hemin/Gq complex as a peroxidase is lower than that of $\mathrm{HRP}^{7}$ and the hemin/Gq complex is easier to be inactivated during the reaction with hydrogen peroxide, ${ }^{8}$ hemin/Gq complexes hold fascinating advantages such as low cost and high thermal stability. Moreover, a wide variety of ssDNA sequences with various chemical modifications as a sensing probe can easily be obtained. They can easily be modified for immobilization on an electrode, and they can be designed as desired to connect functional domains. Also there are many molecular-biological tools to handle them. Therefore, this review briefly surveys the use of hemin/Gq complexes as electrochemical signal generators for the detection of bioanalytes. The electrochemical methods used to detect hemin/Gq complexes are summarized in Section 2. Approaches for amplifying the signal are discussed in Section 3. In addition to traditional enzymatic redox recycling, DNA-specific techniques are introduced to increase the number of signal-generating hemin/Gq complexes. Although hemin/Gq complexes work as signal generators, they do not have the ability to target specific bioanalytes. Thus, the targeting strategies for electrochemical biosensors with hemin/Gq complexes are reviewed in Section 4. Finally, the feasibility of using hemin/Gq complexes to analyze cellular function is discussed.

\section{Electrochemical Detection of Hemin/Gq Complexes}

\subsection{Amperometric detection of hemin bound to $\mathrm{Gq}$}

Hemin that is complexed with $\mathrm{Gq}$ can be placed on the surface of an electrode and directly measured by electrochemical assay. For example, thiol-modified DNA oligomers with guanine-rich sequences are self-assembled onto the surface of a gold electrode, forming a hemin/Gq complex. Also in many cases, target recognition facilitates the attachment of hemin/Gq complex onto 
(a)

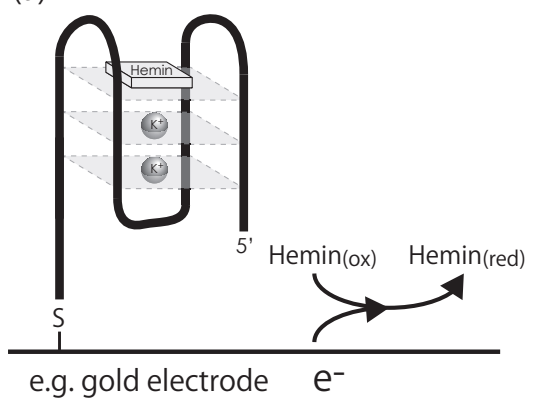

(b)

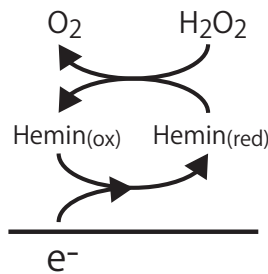

(c)

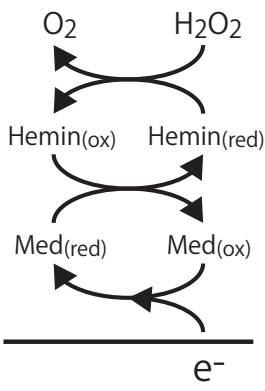

Figure 2. Schematic for the amperometric detection of a hemin/Gq complex on the surface of a gold electrode. (a) Direct reduction of hemin. (b) Reduction of hemin with hydrogen peroxide recycling. (c) Mediator-based detection.

the electrode like Enzyme-Linked ImmunoSorbent Assay (ELISA) or target recognition directs the autonomous folding of the guaninerich sequence into the $\mathrm{Gq}$ structure that binds to hemin (please see section 4.1 and section 4.2 for their targeting strategies). Consequently, the amount of hemin bound to Gq on the surface of the electrode is proportional to the amount of target. Typically the amount of bound hemin was directly measured [Fig. 2(a)] by differential pulse voltammetry (DPV) and a cathodic peak current appeared at around -340 to $-380 \mathrm{mV}$ vs. a saturated calomel electrode (SCE) ${ }^{9-11}$

A hemin/Gq complex can catalyze the reduction of hydrogen peroxide, whereby the complexed hemin is oxidized. On an electrode, this hemin can be subsequently reduced to its original form. The hemin/Gq complex can then be re-oxidized if there is a sufficient amount of hydrogen peroxide. This cycling reaction amplifies the cathodic current, generating an electrochemical signal [Fig. 2(b)]. Cathodic peaks attributed to hemin are generally observed around $-410 \mathrm{mV}$ vs. SCE by cyclic voltammetry $(\mathrm{CV}) ;{ }^{12,13}$ however, lower potentials $(-600 \mathrm{mV}$ to $-800 \mathrm{mV}$ vs. SCE) have also been selected to obtain clear current-response signals. ${ }^{14-16}$

\subsection{Mediator-based amperometric detection}

Electron mediators can enhance the efficiency of electron transfer between hemin and the electrode while avoiding the effects of dissolved oxygen; these characteristics improve the sensitivity of the hemin/Gq based sensor. It was previously reported that electron mediators traditionally used for an enzyme electrode could also work with hemin/Gq-based peroxidase [Fig. 2(c)]. Ferrocene, methylene blue, and toluidine blue, which are typical electron mediators for HRP and glucose oxidase (GOD), ${ }^{17-19}$ were successfully used as mediators for hemin/Gq; they yielded cathodic peaks at around $+360 \mathrm{mV}$ vs. SCE [measured by DPV with a glassy carbon electrode $(\mathrm{GCE})^{20}$ ], around $-210 \mathrm{mV}$ vs. SCE [measured by DPV with a gold nanoparticle (AuNP)-modified $\mathrm{GCE}^{21}$ ], and around $-280 \mathrm{mV}$ vs. SCE (by DPV with a AuNP-modified $\mathrm{GCE}^{22}$ ), respectively. Hydroquinone also works as a redox mediator, producing a cathodic peak at around $-10 \mathrm{mV}$ vs. SCE (by DPV with a AuNP-modified gold electrode ${ }^{23}$ ). The colorimetric substrate for an HRP assay, 3,3,5,5'-tetramethylbenzidine sulfate (TMB), is also oxidized by the hemin/Gq complex with hydrogen peroxide and functions as an electron mediator. The cathodic current of oxidized TMB is around $+320 \mathrm{mV}$ vs. $\mathrm{Ag} / \mathrm{AgCl}$ (measured by DPV with a gold electrode ${ }^{24}$ ).

\subsection{Electrochemical impedance spectroscopy (EIS)}

The surface modification of an electrode and the conformational change of guanine-rich nucleic acids forming a hemin/Gq complex on the electrode can be evaluated by EIS with $\mathrm{Fe}(\mathrm{CN})_{6}{ }^{3-/ 4-}$ as a redox compound. Typically, the immobilization of thiol-modified guanine-rich nucleic acids on a gold electrode utilizing the thiolgold interactions increases charge transfer resistance $R_{\mathrm{ct}}$ relative to a bare electrode because the negatively charged phosphate backbone of the nucleic acids electrostatically hinders $\mathrm{Fe}(\mathrm{CN})_{6}{ }^{3-/ 4-}$ from approaching the electrode surface. The surface modification of hemin/Gq on the gold electrode further increased the $R_{\mathrm{ct}}$ value despite the electroactivity of the iron-containing porphyrin. ${ }^{9}$ Presumably, the hemin/Gq complexes formed an orderly, dense, negatively charged layer that coulombically repelled $\mathrm{Fe}(\mathrm{CN})_{6}{ }^{3-/ 4-}$ from the electrode surface. ${ }^{11}$

EIS is also employed as a sensing principle to evaluate hemin/Gq peroxidase activity. For example, $\alpha$-naphthol was catalytically oxidized to 1,4-naphtoquinone by the hemin/Gq complex in the presence of hydrogen peroxide. The oxidized product precipitated onto the DNA-modified electrode, increasing $R_{\mathrm{ct}}$ and enabling the indirect detection of the concentration of $\alpha$-naphthol by EIS. ${ }^{25}$ Another report has used hemin/Gq and aniline to detect microRNA (miRNA) by EIS. ${ }^{26}$ The anionic miRNA strands captured on an electrode surface induce aniline molecules on the surface with strong electrostatic attraction. The aniline molecules are then catalytically polymerized by the hemin/Gq complex in the presence of hydrogen peroxide. The resulting polyaniline deposits onto the electrode and behaves as an electrical insulator in an alkaline medium, leading to a large increase in $R_{\mathrm{ct}}$ that indirectly reflects the concentration of miRNA.

\subsection{Potentiometry}

Potentiometry is another technique that has been applied to the measurement of hemin/Gq peroxidase activity. It was found that an electrode made from a membrane of tridodecylmethylammonium chloride (TDMAC) plasticized with di- $n$-octyl phthalate (DOP) exhibits highly sensitive potential responses against electrically neutral oligomeric phenols, while the responses towards monomeric phenolic substrates are rather low. The hemin/Gq complex was found to catalyze the oligomerization of monomeric phenols in the presence of hydrogen peroxide, and thus the catalytic activity was evaluated by a potentiometric measurement with the TDMAC-DOP membrane electrode. ${ }^{27}$

\section{Signal Amplification Methods}

\subsection{Enzymatic recycling of hemin redox}

The cathodic current of a biosensor can be amplified by recycling oxidized hemin; for example, an excess of hydrogen peroxide can be used to drive the reaction of hemin/Gq-based peroxidase [Fig. 2(b)]. Instead of directly using hydrogen peroxide, enzymes can amplify the signal of hemin/Gq-based electrochemical detection [Fig. 3(a)]. For example, GOD, a well-known enzyme that catalyzes glucose oxidation and produces hydrogen peroxide, was co-immobilized on a graphene-based scaffold with hemin/Gq 
(a)

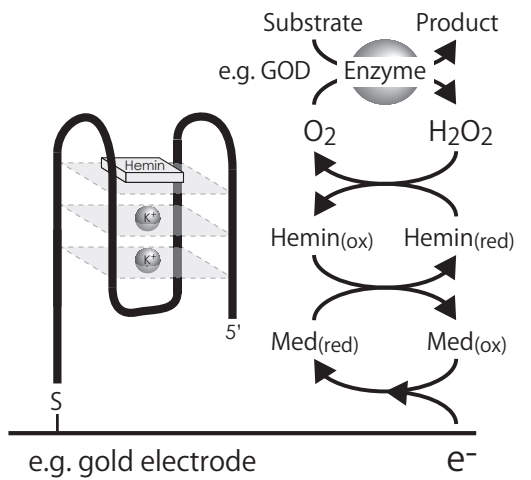

(b)

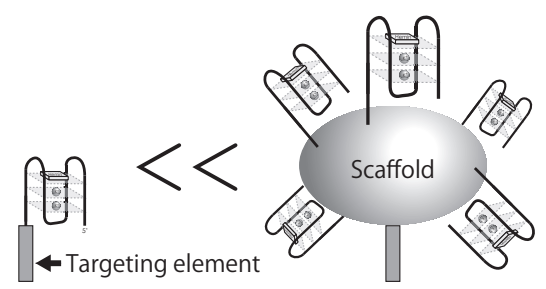

(c)

(1)

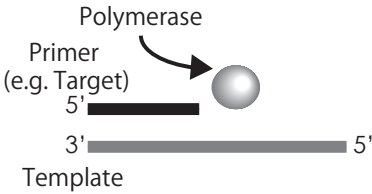

(2)
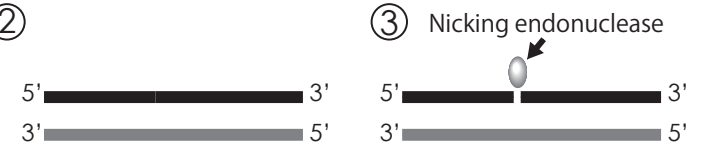

(4)

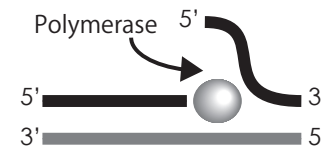

returen to (2)

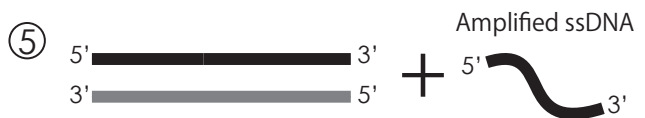

(d)

(1)

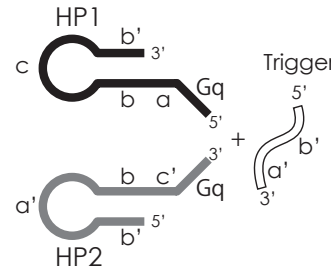

(2)

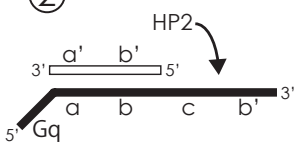

(3)

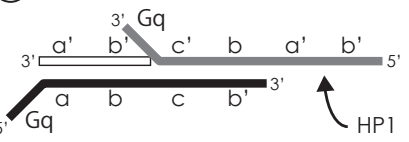

(4)

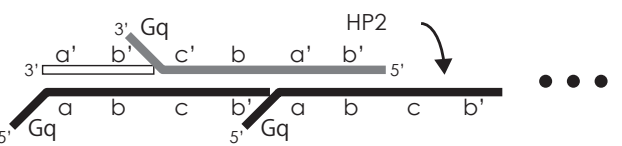

Figure 3. Schematic of the signal amplification methods. (a) Enzymatic recycling of hemin redox. (b) An example of a scaffold-based method to increase the number of hemin/Gq complexes. (c) The basic principle of isothermal amplification of ssDNA. (d) The basic principle of HCR forming a concatamer to increase the number of Gq sequences.

complexes, electron mediators, and targeting elements. This hybridized scaffold was used as an amplified electrochemical signaling tag in the presence of glucose. ${ }^{22} \mathrm{Hemin} / \mathrm{Gq}$ has been found to behave like nicotinamide adenine dinucleotide (NADH) oxidase as well as functioning as a peroxidase ${ }^{28}$ thus, enzymes that utilize NADH as a cofactor have been used to construct a cascade for enzymatic signal amplification. An alcohol dehydrogenase and a glucose dehydrogenase oxidize their substrates and generate NADH. NADH is then oxidized by oxygen in solution through the catalytic action of hemin/Gq as a NADH oxidase, generating $\mathrm{NAD}^{+}$and hydrogen peroxide. In this instance, hydrogen peroxide behaves as a substrate for hemin/Gq-based peroxidase, amplifying the electrochemical signal directly ${ }^{29,30}$ or via a redox mediator. $^{21}$

\subsection{Increasing the number of hemin/Gq complexes}

To enhance an electrochemical signal, a simple approach is to increase the number of signal generators. This section discusses three different methods that increase the number of hemin/Gq complexes.

\subsubsection{Scaffold-based increase}

Synthetic DNA is a popular nanomaterial because it can be tailored via a wide range of chemical modifications. Biotinylation was used to covalently anchor biotin onto DNA, generating a streptavidin-biotinylated DNA conjugate. Against a target, the conjugate increased the number of hemin/Gq complexes by more than a factor of two ${ }^{16}$ because streptavidin is a protein that has four biotin-binding domains. Thiol modification of the ssDNA enabled Gq sequences to self-assemble onto AuNP scaffolds; this increased the number of bound hemin/Gq complexes [Fig. 3(b)] and dramatically enhanced the electrochemical signals. ${ }^{23,31-34}$ In many cases, AuNPs were used to immobilize hemin/Gq on the solid surface of a GCE. Nanocomposites, such as porous platinum nanotubes, ${ }^{29}$ platinum-palladium nanowires,${ }^{30}$ and palladium nanoparticles decorated with graphene-molybdenum disulfide, ${ }^{23}$ were also used as scaffolds to increase the number of hemin/Gq complexes, facilitating co-immobilization of other components, such as enzymes and electron mediators. These scaffolds are donated as a signal generating tag for the simple targeting strategy (please see section 4.1). 


\subsubsection{Isothermal amplification using an enzyme}

Several newly discovered polymerases can efficiently displace synthesized strands, which enables the isothermal amplification of ssDNA without complicated temperature control. The combination of Bst polymerase and a nicking endonuclease, Nt.Bst $\mathrm{NBI}$, has been used to amplify a specific length of ssDNA; this process is an isothermal exponential amplification reaction (EXPAR). The template-guided polymerase reaction (often the primer is a target) generates double-stranded DNA (dsDNA), including a sequence that is recognized by the nicking endonuclease. The products are cleaved by the nicking enzyme. The polymerase begins to repolymerize from the nicking site and the pre-existing strand is displaced as a free, amplified ssDNA [Fig. 3(c)]. Here, if the ssDNA contains the primer sequence in its $3^{\prime}$ end, the amplified ssDNA can hybridize to another template, working as a new primer for another isothermal amplification reaction. Thus the amplification goes exponentially. The resulting ssDNA can be either a direct $\mathrm{Gq}$ sequence $^{35}$ as materials for a concatamer-based signal generating tag (section 3.2.3) or trigger strands that remove an inhibition of autonomous formation of a hemin/Gq complex ${ }^{24,36}$ (section 4.2). In both cases, the number of active hemin/Gq complexes is successfully increased in isothermal conditions. Rolling circle amplification (RCA) is an alternate isothermal amplification strategy that employs Phi29 polymerase to produce long sequences of ssDNA. Phi29 polymerase uses a primer to initiate polymerization of the complementary DNA sequence on a circular template. When the polymerase meets the product, which is hybridized on the circular template, the polymerase undergoes a second round of polymerization on a new segment of the strand that is coupled to the pre-existing segment which detaches. The resulting strand contains the tandem sequence that is complementary to the circular template. RCA has successfully increased the number of hemin/Gq complexes on an electrode under isothermal conditions, yielding high electrochemical sensitivity. ${ }^{37,38}$

\subsubsection{Concatamers}

A DNA concatamer is a polymerized dsDNA structure formed from short ssDNA fragments that self-assemble in a repeating sequence. The formation of a DNA concatamer provides an isothermal approach to increasing the number of hemin/Gq complexes. A simple concatamer-forming pair (one component had a Gq sequence and the other was modified with ferrocene) successfully introduced a long dsDNA tag, which comprised many hemin/Gq complexes with ferrocene as an electron mediator, onto an ssDNA-modified antibody. ${ }^{20}$ The trigger-based formation of concatamers with hairpin (HP)-structured ssDNA fragments is called a hybridized chain reaction (HCR); it has also been employed to achieve concatamer-based amplification [Fig. 3(d)]. Two HP DNA strands (HP1 and HP2) are needed to perform an HCR; these HPs are stable in the absence of a trigger strand. When added, the trigger strand initiates partial hybridization with HP1, revealing the un-hybridized part of the strand as a new trigger for HP2. Similarly, HP2 hybridizes with the newly formed trigger forming the doublestranded concatamer. The un-hybridized part of HP2 becomes a trigger for HP1; thus, the isothermal chain reaction continues. By designing the HP sequences to contain Gq, HCR-based concatamers with tandem hemin/Gq complexes were able to achieve ultrasensitive detection. . $^{21,24,35,39}$

\section{Targeting strategy}

\subsection{Simple targeting}

Although the hemin/Gq complex is electroactive and exhibits enzymatic activity, these functions are not target-specific. The addition of a target recognition element as a functional domain, such as an antibody or an aptamer, to the hemin/Gq complex is a simple way to achieve targeting [Fig. 3(b)]. Immunoassay is one of the most versatile methods for analyzing bioanalytes based on the ability of an antibody to recognize a specific target. In conventional electrochemical immunoassays, enzymes, such as HRP and alkaline phosphatase, are chemically labeled on the antibody and used as signal generators. Similarly, the hemin/Gq complex has been used as a signal generator for antibody labeling. For instance, prostatespecific antigen (PSA) was detected with a streptavidin-conjugated antibody labeled with a biotinylated $\mathrm{Gq}$ sequence. ${ }^{16}$ The detection limit was $0.14 \mathrm{ng} / \mathrm{mL}$. In addition, $0.1 \mathrm{pg} / \mathrm{mL}$ of $\operatorname{IgG} 1$ was measured with concatamer-based signal amplification to demonstrate the generality of the method. ${ }^{20}$ DNA aptamers are oligonucleotides that bind to a specific target. An ssDNA probe can be designed by coding an aptamer sequence connected to a Gq sequence; this method is similar to an immunoassay with an HRPconjugated antibody. Aptamers can be screened from a large pool of random sequences and targets range from small molecules to living cells. Thrombin (with the detection limits of $60 \mathrm{pM},{ }^{14} 0.0067 \mathrm{pM},{ }^{30}$ and $\left.0.1 \mathrm{pM}^{40}\right)$, Escherichia coli $(8$ colony forming units $/ \mathrm{mL}),{ }^{38}$ and human liver hepatocellular carcinoma cells $(30 \text { cells } / \mathrm{mL})^{33}$ have all been electrochemically detected with an aptamer connected to a $\mathrm{Gq}$ sequence. These experimental results and the flexibility of aptamer selection both indicate that aptamer-based targeting has the potential to become a versatile analytical method for hemin/Gq based electrochemical assays.

\subsection{Inhibitor removal strategy}

Guanine-rich sequences have the ability to autonomously bind to hemin, forming a Gq structure and exhibiting peroxidase activity. Inhibiting the autonomous assembly of the hemin/Gq complex can prevent it from exhibiting peroxidase activity. Therefore, the targetspecific removal of inhibiting factors revives the peroxidase activity of hemin/Gq, generating a target-specific signal [Fig. 4(a)]. For example, a stem-loop structure (in this case, an HP) has been developed such that the stem is dsDNA composed of a Gq sequence [Fig. 4(b)]; this structure does not exhibit peroxidase activity under normal conditions. When a target hybridizes to the loop with a longer matching sequence than the stem, the stem is pulled apart, revealing ssDNA that can autonomously form a hemin/Gq complex. Using this strategy, miRNA ${ }^{23}$ and a partial DNA sequence of human immunodeficiency virus type $1(\mathrm{HIV}-1)^{41}$ have been targeted with the detection limits of $0.006 \mathrm{pM}$ and $54 \mathrm{pM}$, respectively.

The key for this strategy is the target-triggered removal of an inhibitor; this creates a free sequence of ssDNA, allowing the autonomous formation of a hemin/Gq complex. Some enzymes, such as a duplex specific nuclease (DSN) or a nicking endonuclease, have been used to remove an inhibitory segment of the hybridized dsDNA. The hybridization of a target nucleic acid to an ssDNA probe that contains a self-inhibition sequence results in a partial sequence of dsDNA. This dsDNA is then treated with either DSN or the nicking endonuclease to digest the hybridized segment, producing a shortened, free ssDNA. Consequently, digestion of the inhibitor site (the dsDNA segment) revives the Gq sequence and enables the formation of the hemin/Gq complex. This strategy has been demonstrated for the successful detection of miRNA $(8 \mathrm{aM}),{ }^{42}$ a short DNA model $(10 \mathrm{fM}),{ }^{9}$ and a partial DNA sequence of the p53 gene $(0.30 \mathrm{fM}){ }^{34}$

The target-triggered EXPAR has also been employed to generate inhibition removal strands that allow the autonomous formation of Gq sequences by hybridizing to the loop part of a stem-loop probe; this strategy has been used to detect miRNA ${ }^{36}$ and an avian influenza A (H7N9) viral DNA model. ${ }^{24}$ The detection limits were $5.36 \mathrm{fM}$ and $9.4 \mathrm{fM}$, respectively.

Basically, the inhibitor removal strategies discussed above are limited for the detection of sequence specific nucleic acids. However, there are a few challenges to detect a target except nucleic acids with the strategy utilizing an aptamer. In many cases, 
(a)
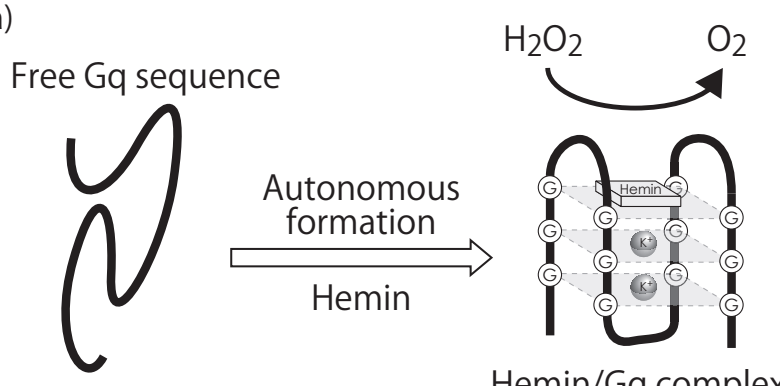

Peroxidase activity ON
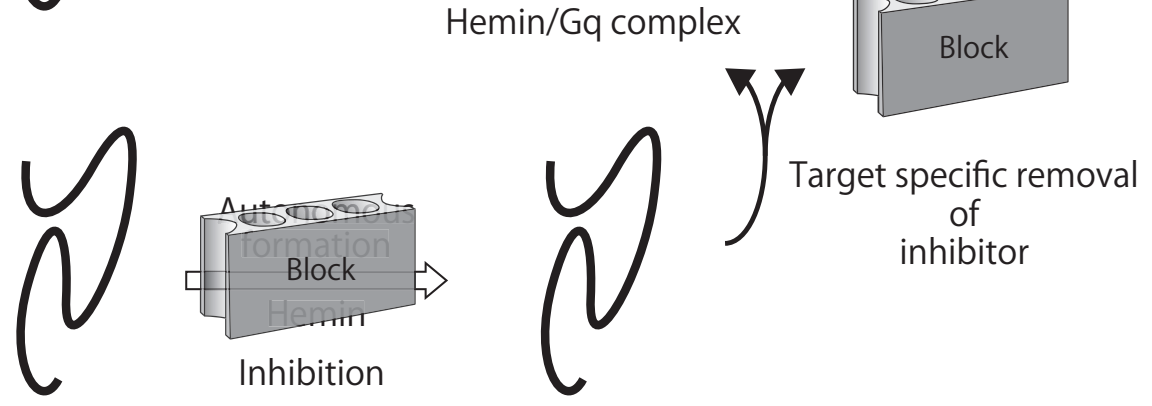

(b)
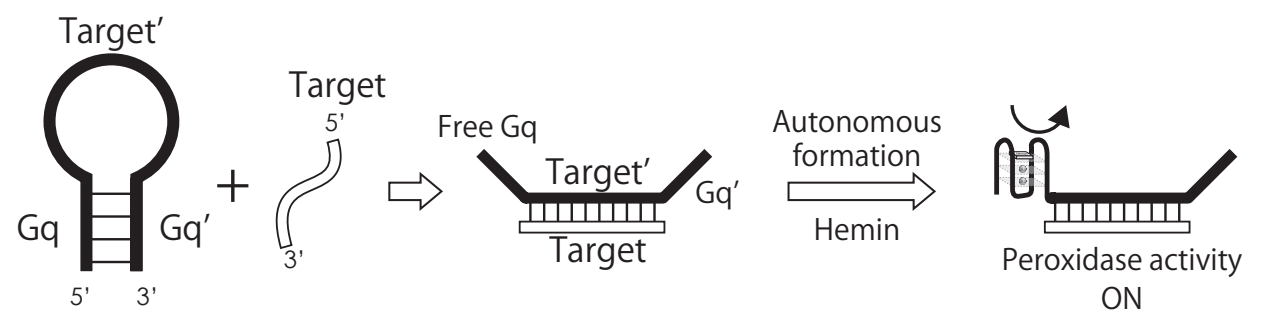

Figure 4. (a) Schematic of the inhibitor-removal strategy to add targeting specificity to a hemin/Gq signal generator. (b) Schematic of an HP-structured hemin/Gq-based peroxidase whose activity is activated by target recognition.

an aptamer changes its conformation when it binds to a target. This dynamic change has been incorporated into the inhibiter removal strategy. An HP probe is designed with a loop composed of an aptamer; when the loop recognizes a target and changes its conformation, the stem of the HP probe is unzipped. The unzipped ssDNA forms a hemin/Gq complex and revives its peroxidase activity. Nucleosides, such as adenosine monophosphate ${ }^{12}$ (AMP), adenosine triphosphate ${ }^{43}$ (ATP), and cytokine protein such as interferon-gamma (IFN- $\gamma)^{15}$ have been successfully detected by this aptamer conformational change with the detection limits of $1 \mu \mathrm{M}$, $10 \mathrm{nM}$, and $0.1 \mathrm{nM}$, respectively. Similarly, the enzymatic activity of adenosine deaminase (ADA), which converts ATP to inosine, has been measured indirectly by ATP aptamer-based hemin/Gq as the signal generator with a detection limit of $0.2 \mathrm{U} / \mathrm{mL} .{ }^{10}$ Although it is not the inhibitor removal strategy, this conformational change of aptamer is also employed to detect a protein utilizing the targettriggered EXPAR. The target recognition of aptamer releases a trigger strand that was originally hybridized to the aptamer. The released trigger strand works as the primer for EXPAR producing direct $\mathrm{Gq}$ sequences that accumulate on the electrode as an HCR-based concatamer. Thirty three femtomolar of thrombin was successfully detected with this strategy. ${ }^{35}$

The inhibitor removal strategy was also applied to measure a concentration of protein reflecting its enzymatic activity. Argonaute 2 is an RNA endonuclease and a core protein of RNA-induced silencing complexes (RISCs). Hybridization of miRNA to an HP structure enabled Argonaute 2 to cleave the miRNA hybridized part and free the $\mathrm{Gq}$ sequence; the $\mathrm{Gq}$ structure formed autonomously, reviving its peroxidase activity. The concentration of Argonaute 2 was successfully measured with a detection limit of $5.02 \mathrm{nM}^{11}$

\subsection{Combination with oxidoreductases}

The hemin/Gq complex exhibits enzymatic activity as a peroxidase and behaves like NADH oxidase, ${ }^{28}$ therefore, it is a suitable component of traditional bienzymatic electrodes. Although the sensitivity was not good yet, the combination of GOD and the hemin/Gq complex has been used as a glucose sensor ${ }^{12}$ [Fig. 3(a)]. Similarly, the combination with alcohol dehydrogenase (ADH) should be able to utilize for the detection of alcohol.

\subsection{Polymerase activity}

The number of hemin/Gq complexes is proportional to the signal strength; therefore, polymerase activity that amplifies the Gq sequences can be independently evaluated. Polymerase activity is introduced as a signal amplification strategy in Section 2; however, the polymerization itself may be of interest to researchers. For example, the activity of telomeres is a tumor biomarker. Telomerase polymerizes an ssDNA sequence with the primer that contains TTAGGG sequence. The polymerized ssDNA contains the tandem repeats of TTAGGG sequence that forms hemin/Gq complexes. In this manner, telomerase activity has been successfully evaluated by measuring hemin/Gq peroxidase activity. The telomerase activity from 500 prostate cancer cells $(\mathrm{LNCaP}) / \mathrm{mL}$ was detected with this method. ${ }^{16}$

\section{Closing Remarks}

The hemin/Gq complex is an electroactive compound that exhibits peroxidase activity and behaves like NADH oxidase; thus, it has been used as a signal generator for the electrochemical detection of bioassays. Although it was reported that the unit activity 
of hemin/Gq as a peroxidase is lower than that of HRP and the hemin/Gquadruplex is easier to be inactivated during the reaction with hydrogen peroxide, hemin/Gq offers some advantages over a protein-based signal generator, such as low cost and high thermal stability. More importantly, a great variety of ssDNA sequences designed as desired with various chemical modifications can easily be purchased as sensing probes. Also the sensitivity of the electrochemical signal can be improved by increasing the number of probes through enzymatic techniques, such as isothermal amplification. In contrast, protein-based probes require chemical or genetic modifications which are difficult to conduct, and it is almost impossible to increase the number of probes through enzymatic techniques. As for targets, DNA-based sensing probes with hemin/Gq complex are particularly good at the detection of sequence specific nucleic acids, and also there are a few challenges to detect targets except nucleic acids using aptamers. The variety of aptamers is still limited, however, it is easy to connect an aptamer sequence (targeting element) with a Gq sequence (signal generator) by simply designing the sequence. Although the applications are still at the basic research level, many targets have been successfully detected with the hemin/Gq complex as a signal generator: bioanalytes ranging from a low molecular weight compound, such as ATP, to macromolecules, such as a diagnostic protein marker, specific nucleic acids including miRNA, or living cancer cells. These bioanalytes are critical to investigations of cellular function. Thus, DNA-based sensing probes that contain hemin/Gq as a signal generator are a promising tool for the electrochemical analysis of cellular function, offering a competent alternative to a conventional protein-based signal generator.

\section{Acknowledgment}

The author would like to thank Enago (www.enago.jp) for the English language review in the first draft.

\section{References}

1. D. Sen and W. Gilbert, Nature, 334, 364 (1988).

2. P. Travascio, Y. Li, and D. Sen, Chem. Biol., 5, 505 (1998).

3. P. Travascio, P. K. Witting, A. G. Mauk, and D. Sen, J. Am. Chem. Soc., 123, 1337 (2001).

4. S. Nakayama and H. O. Sintim, Mol. BioSyst., 6, 95 (2010)

5. Y. Xiao, V. Pavlov, R. Gill, T. Bourenko, and I. Willner, ChemBioChem, 5, 374 (2004).

6. B. T. Roembke, S. Nakayama, and H. O. Sintim, Methods, 64, 185 (2013).

7. T. Li, E. Wang, and S. Dong, Chem. Commun., 5, 580 (2009).

8. X. Yang, C. Fang, H. Mei, T. Chang, Z. Cao, and D. Shangguan, Chemistry, 17,
14475 (2011).

9. S. Liu, C. Wang, C. Zhang, Y. Wang, and B. Tang, Anal. Chem., 85, 2282 (2013).

10. K. Zhang, X. Zhu, J. Wang, L. Xu, and G. Li, Anal. Chem., 82, 3207 (2010).

11. N. Yang, Y. Cao, P. Han, X. Zhu, L. Sun, and G. Li, Anal. Chem., 84, 2492 (2012).

12. G. Pelossof, R. Tel-Vered, J. Elbaz, and I. Willner, Anal. Chem., 82, 4396 (2010).

13. I. Kubo, T. Eguchi, Y. Hoshino, M. Liu, H. Abe, and T. Ito, ECS Trans., 50, 1 (2013).

14. B. Shen, Q. Wang, D. Zhu, J. Luo, G. Cheng, P. He, and Y. Feng, Electroanalysis, 22, 2985 (2010).

15. H. Zhang, B. Jiang, Y. Xiang, Y. Chai, and R. Yuan, Analyst, 137, 1020 (2012).

16. J. Liu, C. Y. Lu, H. Zhou, J. J. Xu, Z. H. Wang, and H. Y. Chen, Chem. Commun., 49, $6602(2013)$

17. I. Willner and E. Katz, Angew. Chem., Int. Ed., 39, 1181 (2000).

18. H. Yao, N. Li, S. Xu, J. Z. Xu, J. J. Zhu, and H. Y. Chen, Biosens. Bioelectron., 21, 372 (2005).

19. Y. Liu, J. Lei, and H. Ju, Talanta, 74, 965 (2008).

20. J. Tang, L. Hou, D. Tang, B. Zhang, J. Zhou, and G. Chen, Chem. Commun., 48, 8180 (2012).

21. Y. Yuan, Y. Chai, R. Yuan, Y. Zhuo, and X. Gan, Chem. Commun., 49, 7328 (2013).

22. P. Jing, H. Yi, S. Xue, Y. Chai, R. Yuan, and W. Xu, Anal. Chim. Acta, 853, 234 (2015).

23. X. Meng, Y. Zhou, Q. Liang, X. Qu, Q. Yang, H. Yin, and S. Ai, Analyst, 138, 3409 (2013).

24. Y. Yu, Z. Chen, W. Jian, D. Sun, B. Zhang, X. Li, and M. Yao, Biosens. Bioelectron., 64, 566 (2015).

25. G. Liang, X. Liu, and X. Li, Biosens. Bioelectron., 45, 46 (2013).

26. H. Deng, W. Shen, Y. Ren, and Z. Gao, Biosens. Bioelectron., 60, 195 (2014).

27. X. Wang, Z. Ding, Q. Ren, and W. Qin, Anal. Chem., 85, 1945 (2013).

28. E. Golub, R. Freeman, and I. Willner, Angew. Chem., Int. Ed. Engl., 50, 11710 (2011).

29. A. Sun, Q. Qi, X. Wang, and P. Bie, Biosens. Bioelectron., 57, 16 (2014).

30. Y. Zheng, Y. Chai, Y. Yuan, and R. Yuan, Anal. Chim. Acta, 834, 45 (2014).

31. Z. Bagheryan, J. Raoof, R. Ojani, and P. Rezaei, Analyst, 140, 4068 (2015).

32. Z. Yang, Y. Zhou, R. Yuan, and Y. Chai, ACS Appl. Mater. Interfaces, 7, 10308 (2015).

33. D. Sun, J. Lu, Z. Chen, Y. Yu, and M. Mo, Anal. Chim. Acta, 885, 166 (2015).

34. Z. Wang, J. Xia, D. Song, F. Zhang, M. Yang, R. Gui, L. Xia, S. Bi, and Y. Xia, Biosens. Bioelectron., 77, 157 (2016).

35. S. Xie, Y. Chai, Y. Yuan, L. Bai, and R. Yuan, Anal. Chim. Acta, 832, 51 (2014).

36. Y. Yu, Z. Chen, L. Shi, F. Yang, J. Pan, B. Zhan, and D. Sun, Anal. Chem., 86, 8200 (2014).

37. Q. Wang, C. Yang, Y. Xiang, R. Yuan, and Y. Chai, Biosens. Bioelectron., 55, 266 (2014).

38. Y. Guo, Y. Wang, S. Liu, J. Yu, H. Wang, Y. Wang, and J. Huang, Biosens. Bioelectron., 75, 315 (2016)

39. M. Xu, Z. Gao, Q. Wei, G. Chen, and D. Tang, Biosens. Bioelectron., 74, 1 (2015).

40. Y. Yuan, G. Liu, R. Yuan, Y. Chai, X. Gan, and L. Bai, Biosens. Bioelectron., 42, 474 (2013).

41. X. Wang, A. Jiang, T. Hou, and F. Li, Anal. Chim. Acta, 890, 91 (2015).

42. X. Zhang, D. Wu, Z. Liu, S. Cai, Y. Zhao, M. Chen, Y. Xia, C. Li, J. Zhang, and J. Chen, Chem. Commun., 50, 12375 (2014).

43. G. Wang, L. Chen, Y. Zhu, L. Wang, and X. Zhang, Electroanalysis, 26, 312 (2014). 